\title{
Contracted Nose after Silicone Implantation: A New Classification System and Treatment Algorithm
}

\author{
Yong Kyu Kim ${ }^{1}$, Seungho Shin ${ }^{2}$, Nak Heon Kang ${ }^{2}$, Joo Heon Kim ${ }^{3}$ \\ ${ }^{1} Y K$ Aesthetic Plastic Surgical Clinic, Seoul; ${ }^{2}$ Department of Plastic and Reconstructive Surgery, Chungnam National University Hospital, \\ Daejeon; ${ }^{3}$ Department of Pathology, Eulji University School of Medicine, Daejeon, Korea
}

Background Silicone implants are frequently used in augmentation rhinoplasty in Asians. A common complication of silicone augmentation rhinoplasty is capsular contracture. This is similar to the capsular contracture after augmentation mammoplasty, but a classification for secondary contracture after augmentation rhinoplasty with silicone implants has not yet been established, and treatment algorithms by grade or severity have yet to be developed. Methods Photographs of 695 patients who underwent augmentation rhinoplasty with a silicone implant from May 2001 to May 2015 were analyzed. The mean observation period was 11.4 months. Of the patients, 81 were male and 614 were female, with a mean age of 35.9 years. Grades were assigned according to postoperative appearance. Grade I was a natural appearance, as if an implant had not been inserted. Grade II was an unnatural lateral margin of the implant. Clearly identifiable implant deviation was classified as grade III, and short nose deformation was grade IV.

Results Grade I outcomes were found in 498 patients (71.7\%), grade II outcomes in 101 (14.5\%), grade III outcomes in $75(10.8 \%)$, and grade IV outcomes in 21 patients $(3.0 \%)$. Revision surgery was indicated for the $13.8 \%$ of all patients who had grade III or IV outcomes. Conclusions It is important to clinically classify the deformations due to secondary contracture after surgery and to establish treatment algorithms to improve scientific communication among rhinoplasty surgeons. In this study, we suggest guidelines for the clinical classification of secondary capsular contracture after augmentation rhinoplasty, and also propose a treatment algorithm.

Keywords Rhinoplasty / Prostheses and implants / Implant capsular contracture
Correspondence: Nak Heon Kang Department of Plastic and Reconstructive Surgery, Chungnam National University Hospital, 282 Munhwa-ro, Jung-gu, Daejeon 35015, Korea

Tel: $+82-42-280-7380$

Fax: +82-42-280-7384

E-mail: nhk488@cnu.ac.kr
No potential conflict of interest relevant to this article was reported.

\section{INTRODUCTION}

Augmentation rhinoplasty using a silicone implant has become one of the most frequently performed aesthetic procedures in Asia $[1,2]$. It usually yields favorable results for both the rhino- plasty surgeon and patient. However, unexpected side effects can occur, such as bleeding, hematoma, infection, cerebrospinal fluid leakage, intranasal adhesion, and postoperative deformities [3]. In addition, while the silicone implant is biocompatible, nontoxic, and chemically stable, it can induce significant capsular 
contracture [2]. Capsular contracture is the development of an abnormal fibrotic capsule associated with the immune response to foreign materials in the human body, especially in the breast $[4,5]$. This immunological reaction can occur in anywhere on the body where artificial materials are used and result in an unfavorable appearance. Most frequently, capsular contracture develops after breast augmentation surgery with a silicone implant. The capsular contracture of breast implants after breast augmentation is well graded by size, shape, and hardness using the fourgrade Baker scale. Moreover, the treatment of breast capsular contracture is well established. Surgical treatment is required for the highest grades of contracture (grades III and IV) [6].

In rhinoplasty, contracture can lead to complications such as a protruding prosthesis, deviated prosthesis, and short nose deformity. Skin hardness, color change, dislocation, and extrusion have been observed in cases of nasal capsular contracture. Furthermore, short nose deformity and nostril show have been found in severe cases. These complications have led patients to be dissatisfied with their cosmetic appearance and to demand more revision operations. The secondary operations vary depending on the patient's condition. Surgery may be required to remove the implants and cartilage grafting may be necessary to rebuild the nasal framework.

Unlike breast capsular contracture, capsular contracture after augmentation rhinoplasty using a silicone implant is not consistently classified. The authors propose a simplified classification of capsular contracture after augmentation rhinoplasty with silicone implants and a treatment algorithm by grade.

\section{METHODS}

This study included 695 patients ( 81 men and 614 women; mean age, 35.9 years) who were treated with augmentation rhinoplasty using only a silicone implant between May 2001 and May 2015 at our 2 clinical institutions. The initial sample included all patients who underwent silicone augmentation rhinoplasty at our institutions. However, patients with other complications, such as infection, skin problems, or implant extrusion, were excluded to limit the variability associated with uncertain factors. Patients were examined regularly following treatment for 3 to 18 months (mean, 11.4 months). Usually, photographs of patients were taken 1 week, 2 weeks, 1 month, 3 months, 6 months, 12 months, and 18 months after the operation. The grade was determined by 2 plastic surgeons by direct observation, physical examination, and photographic analysis. The authors identified the patient's skin condition, softness, implant deviation, and other complications. Two surgeons reviewed each other's results to minimize subjective differences between the observers.

\section{New classification system of nasal capsular contracture}

In our classification of nasal capsular contracture (Table 1), grade I represents an ideal result, a perfect outcome without any detectable capsular contracture or implant. No skin discoloration, rigidity, or irregularity was present (Fig. 1A).

Table 1. Classification of nasal capsular contracture after augmentation rhinoplasty

Grade I Natural appearance. No skin color change, hardness, or irregularity.

Grade II Minimal contracture. Unnatural lateral margin and look, but the appearance is acceptable to the patient.

Grade III Moderate contracture. Implant deviation with skin hardness and irregularity.

Grade IV Severe contracture. Contracted nose and short nose deformity with nostril show.

\section{Fig. 1. Gross photographs according to classification grade}

(A) Grade I (natural appearance). (B) Grade II (unnatural lateral implant margin). (C) Grade III (implant deviation). (D) Grade IV (short nose deformation).
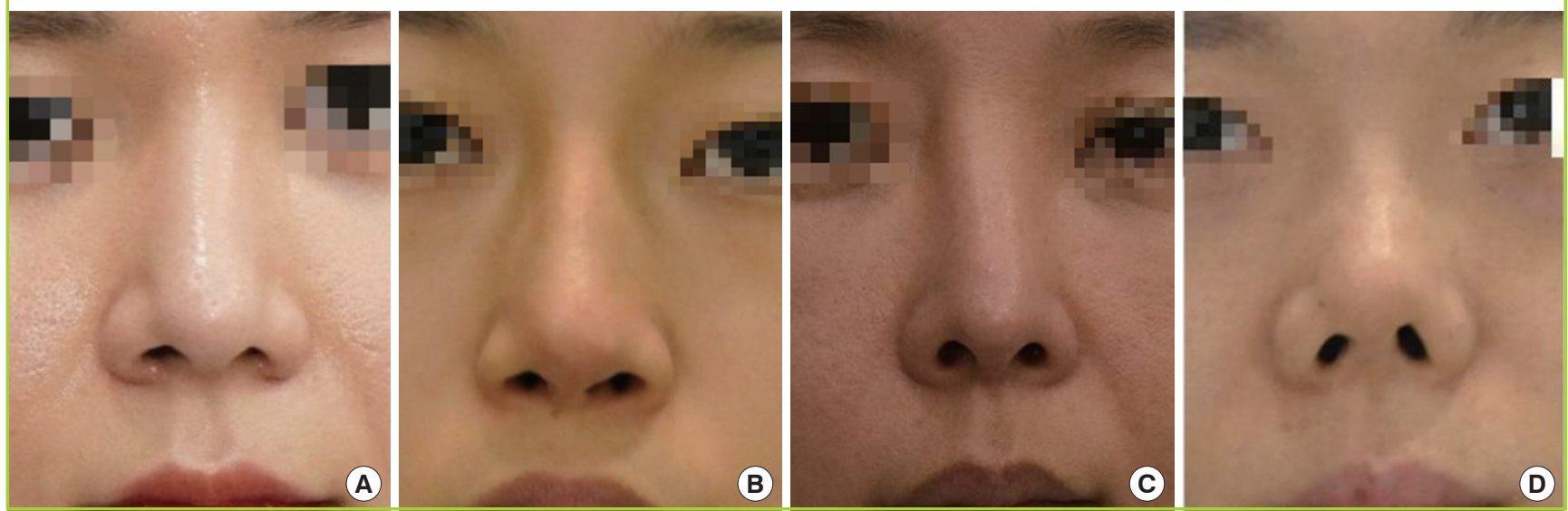
Grade II represents a fair result, but with the implant detectable by the surgeon, suggesting some degree of contraction of the surrounding scar capsule. The margin of the silicone implant was observed by using direct observation and photographic analysis. Some rigidity in the skin and soft tissue was observed in physical examinations. However, the degree of contracture was generally mild and would be unlikely to diminish the patient's satisfaction (Fig. 1B).

In grade III, the degree of capsular contracture was moderate, and the silicone implant was deviated. Patients in this grade were able to recognize the deviation. When surgeons examined the patients, the original softness of the silicone implant had disappeared and implant had developed a hardened and distorted form (Fig. 1C).

Grade IV patients had severe contracture, accompanied by short nose deformity. The short nose deformity caused a reduction in the distance from the nasofrontal angle to nasal tip. The secondary capsular contracture could turn the nasal base upward and cause more nostril show (Fig. 1D).

\section{Surgical methods}

The authors performed the surgical procedures on all grade III and IV patients under local anesthesia and sedation. Following the previous scar, a transcolumellar incision was performed, and the nasal dorsum and radix were dissected subcutaneously. The dissection was performed with scissors to separate the skin flaps. The fibrofatty layer (nasal superficial musculoaponeurotic system, SMAS) was gently retracted with forceps while being elevated with blunt dissection using Iris scissors (Fig. 2).

The surgical technique depended on the patient's condition. In patients with grade III contracture (implant deviation), capsulotomy of the nasal dorsum was performed carefully, followed

\section{Fig. 2. Intraoperative photographs of nasal SMASectomy}

(A) Removal of the nasal superficial musculoaponeurotic system (SMAS) through open rhinoplasty incision. (B) Nasal capsules were held with the forceps and harvested.
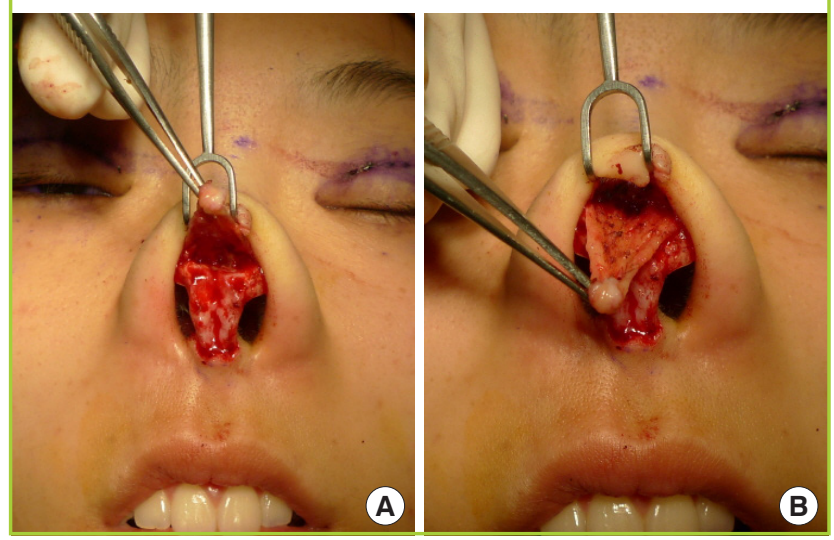

by replacement of the silicone implant. Any scar tissue was removed and released in the nasal tip area. In patients with grade IV contracture, a septal extension graft was required to support the framework. The cartilage was harvested from the ear or nasal septum. Since the patients had previously undergone cartilage graft surgery, if the cartilage was depleted, rib cartilage was used as a graft material. For nasal lengthening and reinforcing the nasal framework, we used techniques such as septal extension grafts, dorsal onlay grafts, and spreader grafts. Alloderm (LifeCell Corporation, Branchburg, NJ, USA) and temporalis fascia were used for a thin skin envelope after capsulectomy.

\section{Capsule harvest and histology}

Capsule specimens were harvested during capsulectomy or capsulotomy procedures performed for revision rhinoplasty in grade III or IV patients. All specimens were harvested from the anterior side of the nasal SMAS in the nasal tip. In the histological preparation, specimens were fixed in $3.7 \%$ formaldehyde solution, embedded in paraffin, cut into $4-\mu \mathrm{m}$ sections with microtomes, and stained with hematoxylin and eosin (H\&E). The $\mathrm{H} \& \mathrm{E}$-stained sections were examined by light microscopy.

\section{RESULTS}

Using our scheme of nasal capsular contracture, we classified the outcomes of 498 patients (71.7\%) as grade I, 101 (14.5\%) as grade II, $75(10.8 \%)$ as grade III, and $21(3.0 \%)$ as grade IV (Fig. 3). In $86.2 \%$ of the patients (grades I and II), revision surgery was not indicated. Grade III patients required capsulotomy for the nasal dorsum, replacement of the silicone, and removal of the thick fibrofatty layer (nasal SMAS) and scar tissue of nasal tip. For grade IV patients, capsulectomy for the nasal dorsum

\section{Fig. 3. Distribution of nasal capsular contracture by grade}

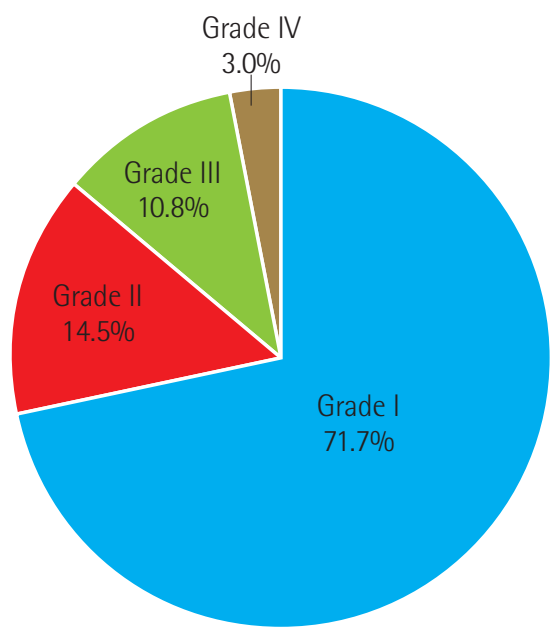


and removal of the fibrofatty layer of the nasal tip were indicated. Severe short nose deformation additionally required a septal extension graft to support the framework. The cartilage was harvested from the ear, nasal septum, or costal cartilage. Alloderm and temporalis fascia were used for a thin skin envelope after capsulectomy (Fig. 4).

Histopathological examinations revealed granulation tissue formation and fibrotic scarring consisting of moderately increased perivascular and interstitial lymphoplasma cells, macrophages, and multinucleated giant cells (Fig. 5). This phenomenon is most often seen in chronic inflammation and foreign body reactions. Our results indicate that the clinical findings regarding capsular contracture and the histological classification grade of inflammatory severity were associated with each other.

\section{Fig. 4. Treatment algorithm for secondary contracture}

Nasal SMAS, nasal superficial musculoaponeurotic system; SEG, septal extension graft.
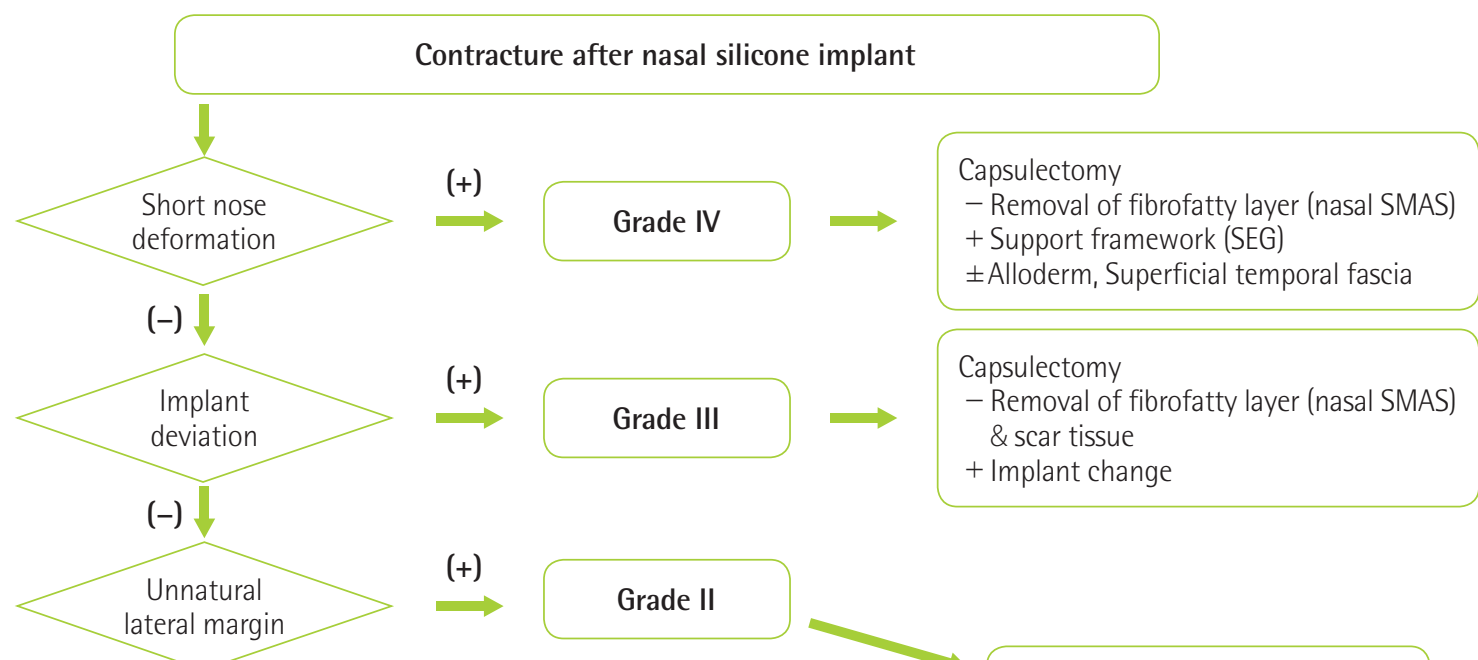

$(+)$
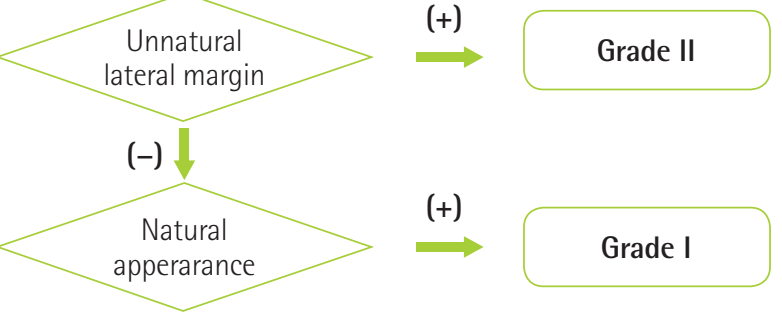

$(+)$

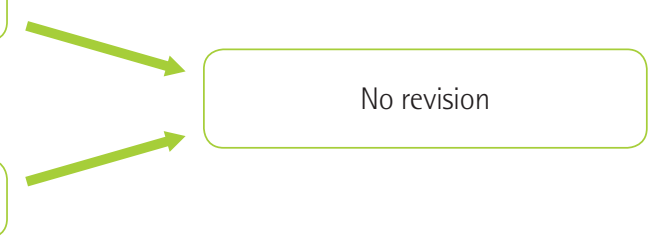

Fig. 5. Pathohistological findings of the nasal capsule

(A) Chronic inflammation (moderately increased perivascular and interstitial lymphoplasma cells and macrophages) is observed, with granulation tissue formation and fibrosis (including a thick fibrous band-like scar) near and surrounding the nasal cartilage. Fragmented cartilage is embedded in the soft tissue $\left(H \& E_{1} \times 100\right)$. (B) Presence of chronic granulomatous inflammation with multinucleated giant cells (foreign body reaction). Presence of foreign-materials $\left(H \& E_{1} \times 400\right)$.
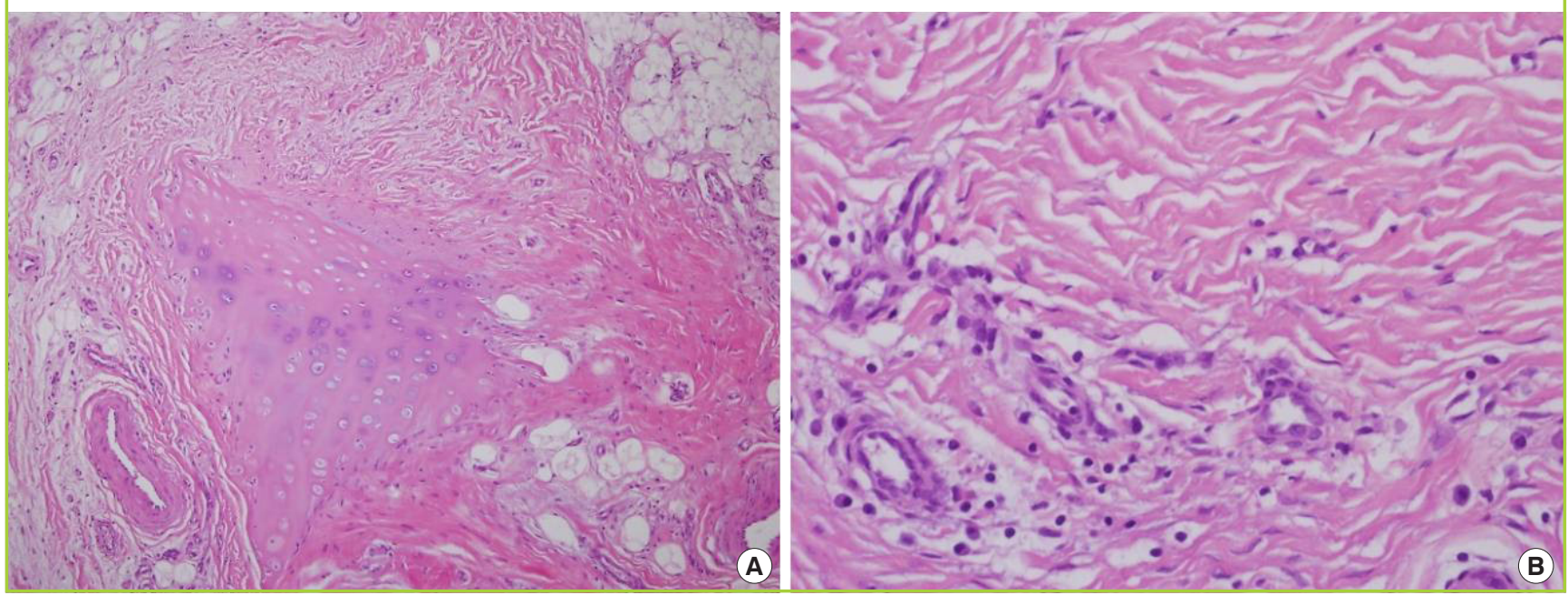


\section{DISCUSSION}

From the perspective of quality of life, cosmetic augmentation rhinoplasty is a surgical procedure with satisfying outcomes. To improve patient satisfaction, highly advanced surgical techniques are being developed, and research to identify an ideal implant material continues. Above all, an ideal implant material must be biocompatible in the human body; that is, it should be eventually resorbed, and maintain its original shape and volume. The implant should be easy to handle, with a high resistance to infection, extrusion, and dislocation, and with minimal foreign body reaction $[7,8]$.

Potential materials for rhinoplasty are abundant. Biological materials have been extensively studied, and include cartilage, fascia, dermis, and combinations thereof. These materials have less infection risk, and are thus preferred by many surgeons, especially for secondary rhinoplasties. Asian noses typically require an augmentation of the dorsum and tip compared to $\mathrm{Eu}$ ropean ethnicities. Softer material, such as fascia and the dermis, might not possess the necessary rigidity. Thus, cartilage is a more appropriate biological alternative. Autologous cartilage is better tolerated and is easy to carve. However, the amount of potential harvest is limited, and unpredictable warping is a major deterrent. Rib cartilage grafts offer enough bulk, but scars at the donor site are often not acceptable. Irradiated cartilage has been used in rhinoplasty, but is not widely available for aesthetic use [9].

Many artificial materials are commonly used in rhinoplasty. These include silicone, Medpor (Porex Surgical, Newnan, GA, USA), Gore-Tex (W.L. Gore \& Associates, Flagstaff, AZ, USA), and AlloDerm (LifeCell Corporation, Branchburg, NJ, USA) [7]. Among these, silicone prostheses are the most popular in Asia, because they are cost-effective, the foreign body reaction is minimal, and they are easy to carve. Nevertheless, potential complications, such as infection, extrusion, contraction, and delayed inflammation, lead to preferences for autologous materials over silicone [10]. The risk of complications after augmentation rhinoplasty with silicone implants has been reported to vary greatly from study to study, from $4 \%$ to $24 \%[8,9,11,12]$.

Complications related to silicone implants, such as discoloration, dislocation, extrusion, contraction resulting from a foreign body reaction, and infection have been reported [12] Among the major complications requiring either removal or revision of the prosthesis, only $2.5 \%$ occurred early, within 30 days of the procedure [9]. In our study, most major complications and any consequent revisions occurred after more than 30 days, but within the first year after the procedure. However, similar to our cases, the reason for most revision operations was not acute inflammation, but cosmetic dissatisfaction [13]. In contrast, delayed complications occurred several years following surgery, and were mainly caused by prosthetics or tissue problems. The delayed contour deformities associated with silicone implants are presumably caused directly by capsular contraction $[14,15]$.Jung et al. [16] proposed that contour changes after silicone augmentation rhinoplasty, including short nose deformities, are related to capsular contracture, pressure necrosis of the lower lateral cartilage, and infections associated with nasal implantation.

Lee et al. [17] reported an implant extrusion rate of $2.1 \%$ to $3.7 \%$, and a displacement rate of $3.7 \%$. According to Tham et al. [9], augmentation rhinoplasty with silicone implants yielded a $16 \%$ complication rate and an $8 \%$ reoperation rate. Extrusion occurred in $2.8 \%$ of cases, through the nasal tip (70\%) or on the posterolateral aspect of the columella (30\%). Deviation occurred in 5.0\% of cases, and other deformities occurred in $2.8 \%$ of cases, and were typically caused by capsular contracture.

In our study, the complication rate requiring reoperation (14.3\%) was slightly higher. This principally occurred because surgeons performed revision surgery at the request of unsatisfied patients, even if prominent deviation or extrusion of the implant did not occur. The rate of mandatory reoperation (2.9\%) due to severe capsular contracture was not high compared to other studies.

The Baker classification of breast capsular contracture remains a stable evaluation system that is in use worldwide. It is a valuable tool for evaluating breast capsular contraction and facilitating the exchange of opinions among surgeons. In some cases, the original form of the Baker classification has been modified for more specific uses, and the advanced classification of breast capsular contracture has been applied to capsular contracture after reconstructive breast operations [18]. In contrast, no definitive criteria like the Baker classification of breast contracture have been proposed for nasal capsular contracture. In this study, we propose a grading system for capsular contracture after nasal silicone implant insertion. Our classification is simple and easy to follow. Such grades of capsular contracture can be useful to assess the postoperative status of patients who have undergone augmentation rhinoplasty and to assess the necessity of revision operations.

In this study, our aims were to provide a clinical classification scheme of the deformations associated with secondary contracture after surgery and to establish treatment algorithms for better scientific communication among rhinoplasty surgeons. Additionally, we suggested guidelines for the clinical classification of secondary capsular contracture after augmentation rhinoplasty with a silicone implant, and proposed a treatment algo- 
rithm. Although our classification of nasal capsular contracture does not directly reflect the severity of contracture, it is associated with the clinical manifestations of capsular contracture. The authors hope this classification encourages more direct communication among rhinoplasty surgeons, and that it will broaden and deepen their understanding of secondary contracture.

\section{REFERENCES}

1. Wu WT. The Oriental nose: an anatomical basis for surgery. Ann Acad Med Singapore 1992;21:176-89.

2. Jung DH, Kim BR, Choi JY, et al. Gross and pathologic analysis of long-term silicone implants inserted into the human body for augmentation rhinoplasty: 221 revision cases. Plast Reconstr Surg 2007;120:1997-2003.

3. Cochran CS, Landecker A. Prevention and management of rhinoplasty complications. Plast Reconstr Surg 2008;122: $60 \mathrm{e}-67 \mathrm{e}$.

4. Granchi D, Cavedagna D, Ciapetti G, et al. Silicone breast implants: the role of immune system on capsular contracture formation. J Biomed Mater Res 1995;29:197-202.

5. Steiert AE, Boyce M, Sorg H. Capsular contracture by silicone breast implants: possible causes, biocompatibility, and prophylactic strategies. Med Devices (Auckl) 2013;6:211-8.

6. Henriksen TF, Fryzek JP, Holmich LR, et al. Surgical intervention and capsular contracture after breast augmentation: a prospective study of risk factors. Ann Plast Surg 2005;54: 343-51.

7. Berghaus A, Stelter K. Alloplastic materials in rhinoplasty. Curr Opin Otolaryngol Head Neck Surg 2006; 14:270-7.

8. Deva AK, Merten S, Chang L. Silicone in nasal augmenta- tion rhinoplasty: a decade of clinical experience. Plast Reconstr Surg 1998;102:1230-7.

9. Tham C, Lai YL, Weng CJ, et al. Silicone augmentation rhinoplasty in an Oriental population. Ann Plast Surg 2005; 54: 1-5.

10. Parker Porter J. Grafts in rhinoplasty: alloplastic vs. autogenous. Arch Otolaryngol Head Neck Surg 2000;126:558-61.

11. Zeng Y, Wu W, Yu H, et al. Silicone implant in augmentation rhinoplasty. Ann Plast Surg 2002;49:495-9.

12. Shirakabe Y, Shirakabe T, Kishimoto T. The classification of complications after augmentation rhinoplasty. Aesthetic Plast Surg 1985;9:185-92.

13. Shirakabe Y, Shirakabe T, Takayanagi S. A new type of prosthesis for augmentation rhinoplasty: our experience in 1600 cases. Br J Plast Surg 1981;34:353-7.

14. Davis PK, Jones SM. The complications of silastic implants: experience with 137 consecutive cases. Br J Plast Surg 1971; 24:405-11.

15. Winkler AA, Soler ZM, Leong PL, et al. Complications associated with alloplastic implants in rhinoplasty. Arch Facial Plast Surg 2012;14:437-41.

16. Jung DH, Moon HJ, Choi SH, et al. Secondary rhinoplasty of the Asian nose: correction of the contracted nose. Aesthetic Plast Surg 2004;28:1-7.

17. Lee MR, Unger JG, Rohrich RJ. Management of the nasal dorsum in rhinoplasty: a systematic review of the literature regarding technique, outcomes, and complications. Plast Reconstr Surg 2011;128:538e-550e.

18. Spear SL, Baker JL Jr. Classification of capsular contracture after prosthetic breast reconstruction. Plast Reconstr Surg 1995;96:1119-23. 